\title{
Pretreatment $A D C$ is not a prognostic factor for local recurrences in head and neck squamous cell carcinoma when clinical T-stage is known
}

\author{
Boris Peltenburg ${ }^{1,2}$ (D) - Juliette P. Driessen ${ }^{3}$. Jeanine E. Vasmel ${ }^{1}$ - Frank A. Pameijer ${ }^{4}$ - Luuk M. Janssen ${ }^{2}$. \\ Chris H. J. Terhaard ${ }^{1} \cdot{\text { Remco de } \text { Bree }^{2} \text { - Marielle E. P. Philippens }}^{1}$
}

Received: 25 April 2019 / Revised: 26 July 2019 / Accepted: 14 August 2019 / Published online: 16 September 2019

(C) The Author(s) 2019

\begin{abstract}
Objectives Pretreatment identification of radio-insensitive head and neck squamous cell carcinomas (HNSCC) would affect treatment modality selection. The apparent diffusion coefficient (ADC) of a tumor could be a predictor of local recurrence. However, little is known about its prognostic value next to known factors such as clinical T-stage. The aim of the present study is to determine the added value of pretreatment ADC to clinical T-stage as a prognostic factor for local recurrence.

Methods This retrospective cohort study included 217 patients with HNSCC treated with (chemo)radiotherapy between April 2009 and December 2015. All patients underwent diffusion-weighted MRI prior to treatment. Median ADC values of all tumors were obtained using a semi-automatic delineation method. Univariate models containing ADC and T-stage were compared with a multivariable model containing both variables.

Results Fifty-eight patients experienced a local recurrence within 3 years. On average, the ADC value in the group of patients with a recurrence was 1.01 versus $1.00\left(10^{-3} \mathrm{~mm}^{2} / \mathrm{s}\right)$ in the group without a recurrence. Univariate analysis showed no significant association between tumor ADC and local recurrence within 3 years after (chemo)radiotherapy $(p=0.09)$. Cox regression showed that clinical T-stage was an independent predictor of local recurrence and adding ADC to the model did not increase its performance.

Conclusion Pretreatment ADC has no added value as a prognostic factor for local recurrence to clinical T-stage.

Key Points

- Pretreatment identification of head and neck squamous cell carcinoma patients who do not benefit from (chemo)radiotherapy could improve personalized cancer care.

- The apparent diffusion coefficient (ADC) obtained from diffusion-weighted MRI has been reported to be a prognostic factor for local recurrence.

- In this study, ADC has no added value as a prognostic factor compared with clinical T-stage.
\end{abstract}

Keywords Diffusion magnetic resonance imaging - Head and neck neoplasms - Neoplasm recurrence, local . (Chemo)radiotherapy $\cdot$ Disease-free survival

Electronic supplementary material The online version of this article (https://doi.org/10.1007/s00330-019-06426-y) contains supplementary material, which is available to authorized users.

Boris Peltenburg

b.peltenburg-3@umcutrecht.nl

1 Department of Radiotherapy, University Medical Center Utrecht, Heidelberglaan 100, 3584 CX Utrecht, The Netherlands
2 Department of Head and Neck Surgical Oncology, UMC Utrecht Cancer Center, University Medical Center Utrecht, Utrecht, The Netherlands

3 Department of Otorhinolaryngology, University Medical Center Utrecht, Utrecht, The Netherlands

4 Department of Radiology, University Medical Center Utrecht, Utrecht, The Netherlands 


\section{Abbreviations}

ADC Apparent diffusion coefficient

AUC Area under the curve

CI95\% 95\% confidence interval

(C)RT (Chemo)radiotherapy

DW-MRI Diffusion-weighted MRI

HNSCC Head and neck squamous cell carcinoma

HPV Human papilloma virus

ROC Receiver operating characteristic

\section{Introduction}

Patients with head and neck squamous cell carcinomas (HNSCC) could benefit from reliable pretreatment identification of radio-insensitive tumors. This would enable patient selection for primary surgery, avoiding the surgical challenges and morbidity induced by previous irradiation [1].

Several studies investigated the correlation of pretreatment apparent diffusion coefficient (ADC) and local tumor recurrence after (chemo)radiotherapy ((C)RT) [2-7]. Most studies concluded that tumors with a relatively high pretreatment ADC have a higher chance of local recurrence [2-4]. However, many of these studies did not account for clinical factors such as T-stage, which is known to be an important prognostic factor for local recurrence [8]. Therefore, the aim of the current study is to find the added value of ADC to clinical T-stage as a prognostic factor for local recurrence of HNSCC.

\section{Methods and materials}

An extended explanation of the methods used in this study can be found in the supplemental materials.

This retrospective study was approved by the institutional review board and the need for informed consent was waived. In total, 217 patients with HNSCC treated with (chemo) radiotherapy were analyzed in the study. Patients were treated between April 2009 and December 2015. All patients underwent diffusion-weighted MRI prior to treatment (Table S1). Available $b$ values differed across scans with all containing at least a high (b800 or $b 1000 \mathrm{~s} / \mathrm{mm}^{2}$ ) and a low $\left(b 0 \mathrm{~s} / \mathrm{mm}^{2}\right) b$ value. Median ADC values of all tumors were obtained using a semi-automatic delineation method. The outcome under investigation, local recurrence, was determined by biopsy $(n=40)$, by progression of a suspected recurrence ( $n=11)$ or by death of the patient during recurrence workup $(n=7)$. All other patients were considered to have local control. Patients were followed up for 3 years. To determine the added value of ADC to clinical T-stage as a prognostic factor for local recurrence, univariable models containing ADC and
T-stage were compared with a multivariable model containing both variables.

\section{Results}

\section{Patients}

A diagram showing the flow of patients is provided in the supplemental material (Fig. S1).

Of the 217 analyzed patients (Table 1), 14 patients $(6.5 \%)$ were lost to follow-up within 3 years. Fifty-eight patients (27\%) developed a local recurrence within 3 years. See S2 for an example of the DW-MRI of one of the patients.

Table 1 Baseline patient characteristics $(N=217)$

\begin{tabular}{|c|c|c|}
\hline Variable & $N$ & $\%$ \\
\hline Age in years & $63(40-87)^{*}$ & \\
\hline \multicolumn{3}{|l|}{ Sex } \\
\hline Female & 55 & 25 \\
\hline Male & 162 & 75 \\
\hline \multicolumn{3}{|l|}{ Tumor site } \\
\hline Larynx & 69 & 32 \\
\hline Hypopharynx & 35 & 16 \\
\hline Oropharynx & 102 & 47 \\
\hline Oral cavity & 11 & 5 \\
\hline \multicolumn{3}{|l|}{ AJCC tumor stage $\mathrm{a}^{\mathrm{a}}$} \\
\hline $\mathrm{T} 2$ & 81 & 37 \\
\hline $\mathrm{T} 3$ & 71 & 33 \\
\hline $\mathrm{T} 4 \mathrm{a}$ & 53 & 24 \\
\hline $\mathrm{T} 4 \mathrm{~b}$ & 12 & 6 \\
\hline \multicolumn{3}{|l|}{ Nodal stage } \\
\hline No & 95 & 44 \\
\hline N1 & 26 & 12 \\
\hline $\mathrm{N} 2 \mathrm{a}$ & 2 & 1 \\
\hline $\mathrm{N} 2 \mathrm{~b}$ & 53 & 24 \\
\hline $\mathrm{N} 2 \mathrm{c}$ & 41 & 19 \\
\hline \multicolumn{3}{|l|}{ HPV status oropharyngeal tumors } \\
\hline Positive & 18 & 18 \\
\hline Negative & 62 & 61 \\
\hline Unknown & 22 & 21 \\
\hline Days between MRI and start treatment* & $18(1-63)$ & \\
\hline \multicolumn{3}{|l|}{ Treatment } \\
\hline Radiotherapy only & 120 & 55 \\
\hline Radiotherapy + cisplatin & 66 & 31 \\
\hline Radiotherapy + cetuximab & 31 & 14 \\
\hline Months of follow-up* & $34(2-102)$ & \\
\hline
\end{tabular}

${ }^{\mathrm{a}}$ AJCC, American Joint Committee on Cancer 7th edition

*Median (range) 


\section{Predictive value of ADC and T-stage for local control}

No significant difference in tumor ADC between the groups with local recurrence and those with local control was found 3 years after $(\mathrm{C}) \mathrm{RT}$ : The mean of median $\mathrm{ADC}$ values $\pm \mathrm{SD}$ was $1.01 \pm 0.18\left(\times 10^{-3} \mathrm{~mm}^{2} / \mathrm{s}\right)$ in the group with local recurrence and $1.00 \pm 0.24\left(\times 10^{-3} \mathrm{~mm}^{2} / \mathrm{s}\right)(p=0.72)$ in the group without local recurrence (S3).

The most discriminating cutoff value of ADC was $0.90 \times$ $10^{-3} \mathrm{~mm}^{2} / \mathrm{s}$. The group of patients with an ADC value higher than this value had no significant difference in the rate of local recurrence compared with the group with $\mathrm{ADC}$ values lower than this value $(p=0.09)(\mathrm{S} 4)$.

The rate of local recurrence was different for each T-stage; patients with $\mathrm{T} 2$ or $\mathrm{T} 3$ tumors had a significantly lower rate of tumor recurrence than the patients with $\mathrm{T} 4 \mathrm{a}$ or $\mathrm{T} 4 \mathrm{~b}$ tumors $(p<0.01)(\mathrm{S} 5)$.

Cox regression showed that T-stage was associated with local recurrence (Table S2). The accuracy of the model expressed as the AUC was 0.66 (CI95\% 0.57-0.74). In the multivariable Cox regression model containing both T-stage and $\mathrm{ADC}$ values, $\mathrm{ADC}$ was not independently associated with local recurrence (Table S3). The AUC of this model was 0.66 (CI95\% 0.58-0.74) while the AUC based solely on ADC was 0.53 (CI95\% 0.45-0.62) (Fig. 1).

\section{HPV and ADC}

Of the 102 patients with oropharyngeal carcinoma, 18 had HPV-positive tumors and 62 were negative for HPV. The
HPV status of the 22 remaining patients was unknown. HPV-positive tumors had a significantly lower ADC value compared with HPV-negative tumors, $0.81 \times 10^{-3} \mathrm{~mm}^{2} / \mathrm{s}$ compared with $0.97 \times 10^{-3} \mathrm{~mm}^{2} / \mathrm{s}(p<0.01)$, respectively. No local recurrences were detected in the HPV-positive group and 18 recurrences occurred in the HPV-negative group. Removing the $18 \mathrm{HPV}$-positive patients from the total sample of 217 patients and retesting the predictive value of ADC and volume did not change the final conclusions.

\section{Importance of findings}

The findings of our study imply that measuring pretreatment ADC does not help clinicians to predict a future local recurrence when T-stage is already known. This is unfortunate as a relatively easily obtainable quantitative tumor characteristic such as pretreatment $\mathrm{ADC}$ would be very helpful in recurrence prediction. The change in ADC $(\triangle \mathrm{ADC})$ between pretreatment $\mathrm{ADC}$ and $\mathrm{ADC}$ values obtained during treatment might have more prognostic value $[5,7,9]$.

\section{Discussion}

In contrast to our study, an association between pretreatment $\mathrm{ADC}$ and local recurrence has previously been described [2-4]. Most of these studies included a substantially lower amount of patients in comparison with our study. Only the study reported by Lambrecht et al [4] had a comparable
Fig. 1 ROC curve displaying the performance of the models containing T-stage and ADC as individual parameters (black and yellow) and the performance of the model containing both variables (blue)
ROC Curve

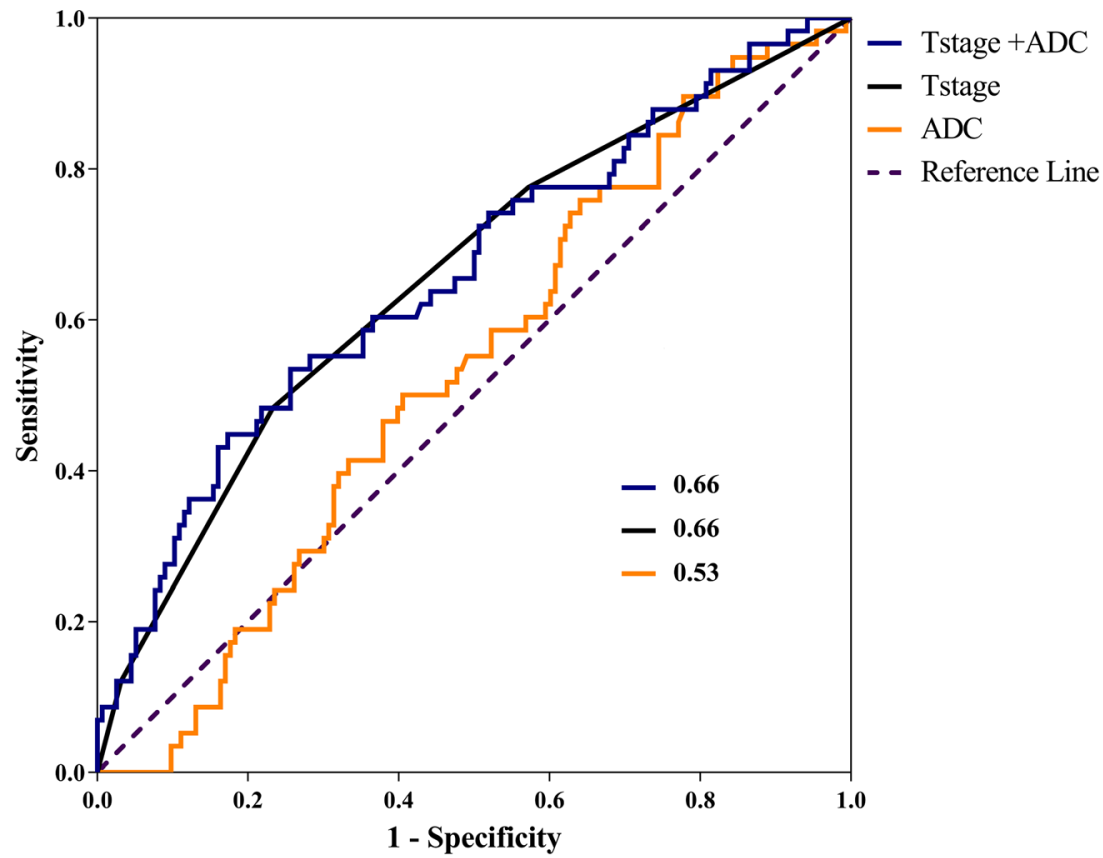


sample size. This study included 161 patients and performed multivariable analysis including, amongst others, tumor ADC and tumor volume. Additionally, similar to our methodology, they created two models, one with ADC included and one without $\mathrm{ADC}$ and compared the performance of the models. They report an AUC, used to determine the discriminatory capacity of the first model, of 0.62 (CI95\% 0.56-0.70), while for the model without ADC, the AUC is 0.60 (CI95\% 0.55$0.67)$. These results are very similar to our findings and it supports our conclusion that ADC has no added value as a prognostic factor for local recurrence to, more easily obtainable, clinical parameters. A full discussion of the results can be found in the supplemental material.

\section{Conclusion}

In this study, we found pretreatment ADC to have no added value to clinical T-stage as a prognostic factor for local recurrences of HNSCC within 3 years after (chemo)radiotherapy.

Acknowledgments We thank A.S. Borggreve for assistance with the statistical analysis.

Funding information The authors state that this work has not received any funding.

\section{Compliance with ethical standards}

Guarantor The scientific guarantor of this publication is M.E.P. Philippens.

Conflict of interest The authors of this manuscript declare no relationships with any companies, whose products or services may be related to the subject matter of the article.

Statistics and biometry A.S. Borggreve kindly provided statistical advice for this manuscript. One of the authors has significant statistical expertise.

Informed consent Written informed consent was waived by the Institutional Review Board.

Ethical approval Institutional Review Board approval was obtained.

\author{
Methodology \\ - retrospective \\ - prognostic study \\ - performed at one institution
}

Open Access This article is distributed under the terms of the Creative Commons Attribution 4.0 International License (http:// creativecommons.org/licenses/by/4.0/), which permits unrestricted use, distribution, and reproduction in any medium, provided you give appropriate credit to the original author(s) and the source, provide a link to the Creative Commons license, and indicate if changes were made.

\section{References}

1. Lee SC, Shores CG, Weissler MC (2008) Salvage surgery after failed primary concomitant chemoradiation. Curr Opin Otolaryngol Head Neck Surg 16:135-140. https://doi.org/10.1097/MOO. 0b013e3282f495b6

2. Hatakenaka M, Shioyama Y, Nakamura K et al (2011) Apparent diffusion coefficient calculated with relatively high b-values correlates with local failure of head and neck squamous cell carcinoma treated with radiotherapy. AJNR Am J Neuroradiol 32:1904-1910. https://doi.org/10.3174/ajnr.A2610

3. Ohnishi K, Shioyama Y, Hatakenaka M et al (2011) Prediction of local failures with a combination of pretreatment tumor volume and apparent diffusion coefficient in patients treated with definitive radiotherapy for hypopharyngeal or oropharyngeal squamous cell carcinoma. J Radiat Res 52:522-530

4. Lambrecht M, Van Calster B, Vandecaveye V et al (2014) Integrating pretreatment diffusion weighted MRI into a multivariable prognostic model for head and neck squamous cell carcinoma. Radiother Oncol 110:429-434. https://doi.org/10.1016/j.radonc.2014.01.004

5. King AD, Mo FK, Yu KH et al (2010) Squamous cell carcinoma of the head and neck: diffusion-weighted MR imaging for prediction and monitoring of treatment response. Eur Radiol 20:2213-2220. https://doi.org/10.1007/s00330-010-1769-8

6. Chawla S, Kim S, Dougherty L et al (2013) Pretreatment diffusionweighted and dynamic contrast-enhanced MRI for prediction of local treatment response in squamous cell carcinomas of the head and neck. AJR Am J Roentgenol 200:35-43. https://doi.org/10.2214/ AJR.12.9432

7. King AD, Chow KK, Yu KH et al (2013) Head and neck squamous cell carcinoma: diagnostic performance of diffusion-weighted MR imaging for the prediction of treatment response. Radiology 266: 531-538. https://doi.org/10.1148/radiol.12120167

8. Brockstein B, Haraf DJ, Rademaker AW et al (2004) Patterns of failure, prognostic factors and survival in locoregionally advanced head and neck cancer treated with concomitant chemoradiotherapy: a 9-year, 337-patient, multi-institutional experience. Ann Oncol 15: $1179-1186$

9. Vandecaveye V, Dirix P, De Keyzer F et al (2010) Predictive value of diffusion-weighted magnetic resonance imaging during chemoradiotherapy for head and neck squamous cell carcinoma. Eur Radiol 20: 1703-1714. https://doi.org/10.1007/s00330-010-1734-6

Publisher's note Springer Nature remains neutral with regard to jurisdictional claims in published maps and institutional affiliations. 